\title{
Detection of Biasing Attacks on Distributed Estimation Networks
}

\author{
Mohammad Deghat
}

\author{
Valery Ugrinovskii
}

\author{
Iman Shames
}

\author{
Cédric Langbort
}

\begin{abstract}
The paper addresses the problem of detecting attacks on distributed estimator networks that aim to intentionally bias process estimates produced by the network. It provides a sufficient condition, in terms of the feasibility of certain linear matrix inequalities, which guarantees distributed input attack detection using an $H_{\infty}$ approach.
\end{abstract}

\section{INTRODUCTION}

With recent rapid developments in the area of networked control and estimation, the security of networked systems against input attacks and faults becomes increasingly important. The mainstream of the results in the literature focus on centralized attack and fault detection, however some recent work has been done on distributed attack and fault detection due to the fact that not all measurements might be available at each node of the network; see [2], [9], [10], [13], [5], [3] and the references therein.

This paper considers the problem of detection of attacks on consensus-based distributed estimation networks. The topic of distributed estimation has gained considerable attention in the literature, in a bid to reduce communication bottlenecks and improve reliability and fidelity of centralized state observers. Filter cooperation and consensus ideas have proved to be instrumental in the design of distributed state observers [7], [15], [16]. At the same time, consensus-based systems are particularly vulnerable to intentional attacks since the compromised agents can interfere with the functions of the entire network in a significant way [8]. Uncertainty and noise represent another challenge from the attack detection viewpoint - state observers are typically required in applications where uncertainty and noise make accessing the system state difficult; this may allow the attackers to remain undetected by injecting signals compatible with the noise statistics [9]. This motivates an increased interest in the literature in detection of rogue behaviours of state observers.

In this paper, we consider a general framework of distributed state estimation considered, for example, in [15], [16], [18] and assume that some of the nodes of the network

This work was supported by the Australian Research Council and the University of New South Wales.

The paper is to appear in Proceedings of the 55th IEEE Conference on Decision and Control, Las Vegas, December 2016.

M. Deghat and V. Ugrinovskii are with the School of Engineering and Information Technology, University of New South Wales at the Australian Defence Force Academy, Canberra, ACT 2600, Australia. m. deghateunsw.edu.au; v.ougrinovski@adfa.edu.au

I. Shames is with the Department of Electrical and Electronic Engineering, University of Melbourne, Melbourne, Victoria 3001, Australia. iman. shameseunimelb.edu.au

C. Langbort is with the Department of Aerospace Engineering and Coordinated Science Laboratory, University of Illinois at Urbana-Champaign, Urbana, IL 61801 USA. langborteillinois.edu are compromised. Mathematically, this situation is modelled by allowing the compromised observers to be driven by certain attack/fault inputs. The purpose of the attack under consideration is to force the compromised node to produce biased state estimates and then exploit the consensus mechanism within the network to propagate those estimates across the network. Conventional false data injections into measurements can also be included in the model as a routine extension of our results.

From the viewpoint of fault detection/input estimation, the system subject to attack is distributed itself. This is similar to [13], but is different from [5], [3] which were focused on detecting faults applied to the observed plant. We use an $H_{\infty}$ fault detection approach which allows for a broad range of uncertainty in the sensors and the plant model, as well as a quite broad range of attack inputs. Furthermore, to detect the attack/fault, the proposed attack observers use the same plant measurements and the state estimate information communicated from the neighbours as the state observers themselves. The key idea is to use this information, without additional communication overheads, to determine which of the node observers' behaviour differs from what this information predicts.

Our idea of governing the detectors by neighbours' state estimates to track the attack input is similar to [12], where integral action controllers governed by diffusive couplings were used for averaging constant disturbances. More precisely, in [12] distributed integral action controllers were used for averaging constant disturbances to enable all agents in the system to synchronize to a common reference system governed by the averaged constant disturbance. In contrast, here we are interested in tracking individual attack inputs, rather than tracking an averaged attack vector. Technically this required us to introduce additional dynamics into the fault detectors. Also unlike [12], the $H_{\infty}$ formulation adopted here does not restrict the attack inputs to be constants.

The paper is organised as follows. In Section II a background on distributed consensus based estimation is presented. Also, the idea of distributed attack estimation with $H_{\infty}$ consensus is explained and the attack detection problem is formulated in that section. The main result is given in Section [III, where a sufficient condition in terms of coupled linear matrix inequalities is expressed. Concluding remarks are given in Section IV

Notation: $\mathbf{R}^{n}$ denotes the real Euclidean $n$-dimensional vector space, with the norm $\|x\|=\left(x^{\prime} x\right)^{1 / 2}$; here the symbol ' denotes the transpose of a matrix or a vector. The symbol $I_{n}$ denotes the $n \times n$ identity matrix, and $0_{m \times n}$ 
denotes the zero matrix of size $m \times n$. We will occasionally use $I$ and 0 for notational convenience if no confusion is expected. For real symmetric $n \times n$ matrices $X$ and $Y$, $Y>X$ (respectively, $Y \geq X$ ) means the matrix $Y-X$ is positive definite (respectively, positive semidefinite). The notation $L_{2}[0, \infty)$ refers to the Lebesgue space of $\mathbf{R}^{n}$-valued vector-functions $z($.$) , defined on the time interval [0, \infty)$, with the norm $\|z\|_{2} \triangleq\left(\int_{0}^{\infty}\|z(t)\|^{2} d t\right)^{1 / 2}$ and the inner product $\int_{0}^{\infty} z_{1}^{\prime}(t) z_{2}(t) d t$.

\section{Formulation of the Distributed ATtACK DETECTION PROBLEM}

\section{A. Network topology}

Consider a filter network with $N$ nodes and a directed graph topology $\mathbf{G}=(\mathbf{V}, \mathbf{E})$ where $\mathbf{V}$ and $\mathbf{E}$ are the set of vertices and the set of edges (i.e, the subset of the set $\mathbf{V} \times \mathbf{V}$ ), respectively. Without loss of generality, we let $\mathbf{V}=\{1,2, \ldots, N\}$. The graph $\mathbf{G}$ is assumed to be directed, reflecting the fact that while node $i$ receives the information from node $j$, this relation may not be reciprocal. The notation $(j, i)$ will denote the edge of the graph originating at node $j$ and ending at node $i$. It is assumed that the nodes of the graph $\mathbf{G}$ have no self-loops, i.e., $(i, i) \notin \mathbf{E}$.

For each $i \in \mathbf{V}$, let $\mathbf{V}_{i}=\{j:(j, i) \in \mathbf{E}\}$ be the set of nodes supplying information to node $i$. The cardinality of $\mathbf{V}_{i}$, known as the in-degree of node $i$, is denoted $p_{i}$; i.e., $p_{i}$ is equal to the number of incoming edges for node $i$. Also, $q_{i}$ will denote the number of outgoing edges for node $i$, known as the out-degree of node $i$. Let $\mathbf{A}=\left[\mathbf{a}_{i j}\right]$ be the adjacency matrix of the digraph $\mathbf{G}$, i.e., $\mathbf{a}_{i j}=1$ if $(j, i) \in \mathbf{E}$, otherwise $\mathbf{a}_{i j}=0$. Then, $p_{i}=\sum_{j=1}^{N} \mathbf{a}_{i j}=\sum_{j \in \mathbf{V}_{i}} \mathbf{a}_{i j}$, $q_{i}=\sum_{j=1}^{N} \mathbf{a}_{j i}$.

B. Background: distributed consensus-based $H_{\infty}$ estimation

A typical distributed consensus-based $H_{\infty}$ estimation problem considers a plant described by the equation

$$
\dot{x}=A x+B_{2} \xi(t), \quad x(0)=x_{0}, \quad x \in \mathbf{R}^{n},
$$

governed by an disturbance input $\xi \in \mathbf{R}^{m}$. A network of filters connected according to the graph $\mathbf{G}$ takes measurements of the plant with the purpose to produce an estimate of $x$. It is assumed that each filter takes measurements

$$
y_{i}=C_{2 i} x+D_{2 i} \xi+\bar{D}_{2 i} \xi_{i},
$$

where $\xi_{i}(t) \in \mathbf{R}^{m_{i}}$ represents the measurement disturbance at the local sensing node $i$, and processes them locally using an information communicated by its neighbours $j, j \in \mathbf{V}_{i}$. Depending on the nature of the disturbances $\xi$, $\xi_{i}$, the processing can be done using Kalman [7] or $H_{\infty}$ [15], [16], [18] filters, both using innovations in the measurements and the neighbours' information for feedback. To be concrete, from now on we build the presentation around the distributed $H_{\infty}$ consensus filter introduced in [16], [18], although the approach to bias attack detection proposed in this paper is general enough to allow extensions to other types of filters in an obvious manner.

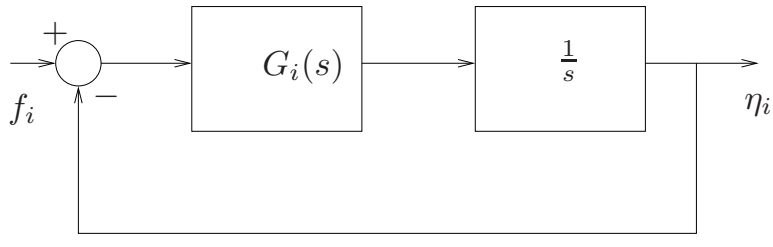

Fig. 1. An auxiliary 'input tracking' model.

According to [16], suppose the disturbances $\xi, \xi_{i}$ belong to $L_{2}[0, \infty)$; this assumption suffices to guarantee that equation (1) has an $L_{2}$-integrable solution on any finite time interval $[0, T]$, even when the matrix $A$ is unstable. Then using the Luenberger type observer, each filter produces an estimate $\hat{x}_{i}$ of the state $x$

$$
\begin{array}{r}
\dot{\hat{x}}_{i}=A \hat{x}_{i}+L_{i}\left(y_{i}(t)-C_{2 i} \hat{x}_{i}\right)+K_{i} \sum_{j \in \mathbf{V}_{i}}\left(\hat{x}_{j}-\hat{x}_{i}\right), \\
\hat{x}_{i}(0)=0,
\end{array}
$$

where the matrices $L_{i}, K_{i}$ are the parameters of the filter. The observer structure indicates that each node takes advantage of being interconnected with other nodes in that each filter uses its neighbours estimates $\hat{x}_{j}, j \in \mathbf{V}_{i}$. The problem in [16] was to determine estimator gains $L_{i}$ and $K_{i}$ in (3) to ensure the filter internal stability and acceptable $H_{\infty}$ attenuation of the effect which disturbances have on the consensus performance of the filter.

\section{The bias attack model}

The particular problem of interest in this paper is to consider the situation where one or several nodes of the network of observers described in the previous sections are subject to bias attack. While a commonly considered situation is when the attacker interferes with the measurements and/or communications between the nodes, here in contrast, we consider the situation where the attacker mounts an attack on the observer dynamics. That is, we consider the situation where in lieu of (3), some of the nodes generate their estimates according to

$$
\begin{array}{r}
\dot{\hat{x}}_{i}=A \hat{x}_{i}+L_{i}\left(y_{i}(t)-C_{2 i} \hat{x}_{i}\right)+K_{i} \sum_{j \in \mathbf{V}_{i}}\left(\hat{x}_{j}-\hat{x}_{i}\right)+f_{i},(4) \\
\hat{x}_{i}(0)=0,
\end{array}
$$

where $f_{i}$ is the attack input. From now on, our focus is exclusively on the network of observers (4).

To present the class of admissible attack signals under consideration in this paper, consider an auxiliary 'input tracking' model shown in Fig. 11 with a stable square $n \times n$ transfer function $G_{i}(s)$, with invertible $G_{i}(0)$. Since $G_{i}(s)$ is square, then input $f_{i}$ and output $\eta_{i}$ of the system in Fig. 1 are of dimension $n$.

Assumption 1: Given a stable square $n \times n$ transfer function $G_{i}(s)$, the class of admissible bias inputs under consideration consists of all signals $f_{i}(t), t \geq 0$, such that

$$
\int_{0}^{\infty}\left\|f_{i}-\eta_{i}\right\|^{2} d t<\infty
$$


Consider the tracking error of the system shown in Fig. 1 $\nu_{i}=\eta_{i}-f_{i}$. Under Assumption 1, $\nu_{i}$ is a finite energy signal. Denoting the Laplace transforms of $f_{i}$ and $\nu_{i}$ as $f_{i}(s)$ and $\nu_{i}(s)$ respectively, and noting that

$$
\nu_{i}(s)=-\left(I_{n}+\frac{1}{s} G_{i}(s)\right)^{-1} f_{i}(s),
$$

condition (5) is equivalent to

$$
\int_{-j \infty}^{+j \infty}\left\|\left(I+\frac{1}{s} G_{i}(s)\right)^{-1} f_{i}(s)\right\|^{2} d s<\infty .
$$

Note also that the invertibility of $G_{i}(0)$ guarantees that $\lim _{t \rightarrow \infty}\left\|f_{i}(t)-\eta_{i}(t)\right\|=0$ for inputs $f_{i}$ that have a finite limit at $\infty$.

In practice, of course the transfer function $G_{i}(s)$ must be selected by the designer based on the anticipated behaviour of the attack inputs $f_{i}(t)$. It remains unknown to the attacker. For example, to capture a class of bias injection attack inputs consisting of a steady-state component and an exponentially decaying transient component generated by a low pass filter [14] it suffices to choose $G_{i}(s)=\frac{1}{s+2 \epsilon_{i}} I_{n}$, where $I_{n}$ is the $n \times n$ identity matrix, and $\epsilon_{i}>0$ is a constant. It must be noted that even with this choice of $G_{i}(s)$, the designer does not need to know the asymptotic steady-state value or the shape of the transient, as all such signals $f_{i}$ satisfy condition (6). Furthermore, such signals have the property that $\lim _{t \rightarrow \infty} f_{i}(t)$ exists and therefore we can ensure that $\left\|f_{i}(t)-\eta_{i}(t)\right\| \rightarrow 0$ as $t \rightarrow \infty$. More generally, signals representing a combination of constants and $L_{2}$-integrable inputs satisfy (6). In addition to bias attack policies $f_{i}$ described above, $L_{2}$-integrable inputs $f_{i}$ are included which represent attack inputs with limited energy resource [14].

It can be readily shown that the state-space model for the system in Fig 1 can be written as

$$
\begin{aligned}
& \dot{\omega}_{i}=\Omega_{i} \omega_{i}+\Gamma_{i} \nu_{i}, \\
& \eta_{i}=\left[\begin{array}{ll}
I & 0
\end{array}\right] \omega_{i}, \quad \omega_{i}(0)=0,
\end{aligned}
$$

where $\nu_{i}=\eta_{i}-f_{i}$ is an $L_{2}$-integrable input, according to Assumption 1. In particular, in the special case $G_{i}(s)=$ $\frac{1}{s+2 \epsilon_{i}} I_{n}$, we have $\omega_{i} \in \mathbf{R}^{2 n}$, and

$$
\Omega_{i}=\left[\begin{array}{cc}
0 & I \\
0 & -2 \epsilon_{i} I
\end{array}\right], \quad \Gamma_{i}=\left[\begin{array}{c}
0 \\
-I
\end{array}\right]
$$

\section{The proposed attack detector}

The objective of the paper is to design a (distributed) attack detection system which is capable of tracking attack inputs satisfying Assumption 1 To this end, we consider the following outputs which summarize the information about the network available at node $i$, and can be used by the attack detector

$$
\begin{aligned}
\zeta_{i} & =y_{i}-C_{2 i} \hat{x}_{i} \\
& =C_{2 i}\left(x-\hat{x}_{i}\right)+D_{2 i} \xi+\bar{D}_{2 i} \xi_{i} \\
\bar{\zeta}_{i} & =\sum_{j \in \mathbf{V}_{i}}\left(\hat{x}_{j}-\hat{x}_{i}\right)
\end{aligned}
$$

The idea behind introducing these outputs is as follows. If node $i$ is under attack, then its predicted sensor measurement $C_{2 i} \hat{x}_{i}$ is expected to be biased, compared to the actual measurement $y_{i}$. This must lead to a significant difference between these two signals, i.e., we must expect a large energy in $\zeta_{i}$. Likewise, the observer under attack is expected to cause the system to deviate from the state of consensus, causing the state of the observer $i, \hat{x}_{i}$ to deviate from the average estimate produced at the neighbouring nodes. Thus, the disagreement variable $\bar{\zeta}_{i}$ at node $i$ is expected to differ from similar variables produced by the rest of the network. This motivates using these outputs for detecting the attack.

Let $e_{i}=x-\hat{x}_{i}$ be the local estimation error at node $i$. Using (1) and (4), it is straightforward to verify that the local filter errors satisfy the following equation:

$$
\begin{aligned}
\dot{e}_{i} & =\left(A-L_{i} C_{2 i}\right) e_{i}+K_{i} \sum_{j \in \mathbf{V}_{i}}\left(e_{j}-e_{i}\right) \\
& +\left(B_{2}-L_{i} D_{2 i}\right) \xi-L_{i} \bar{D}_{2 i} \xi_{i}-f_{i}, \quad e_{i}(0)=x_{0}
\end{aligned}
$$

The outputs (9), (10) can be rewritten in terms of the estimation errors as

$$
\begin{aligned}
& \zeta_{i}=C_{2 i} e_{i}+D_{2 i} \xi+\bar{D}_{2 i} \xi_{i}, \\
& \bar{\zeta}_{i}=-\sum_{j \in \mathbf{V}_{i}}\left(e_{j}-e_{i}\right) .
\end{aligned}
$$

Hence, we can consider the collection of systems (11) as a large-scale plant governed by the vector of attack inputs $f=\left[f_{1}^{\prime}, \ldots, f_{N}^{\prime}\right]^{\prime}$, and equipped with the outputs (12), 13. It is worth stressing that these outputs can be readily generated at the observer $i$, computing them only requires the local measurements $y_{i}$, the local estimate $\hat{x}_{i}$ computed by the observer at node $i$ and the neighbours estimates $\hat{x}_{j}, j \in \mathbf{V}_{i}$, available to that observer. Therefore the outputs (12), (13) are available for tracking the attack inputs. To achieve this, consider the system combining the estimation error dynamics (11) and the auxiliary input tracking model (7):

$$
\begin{aligned}
\dot{e}_{i} & =\left(A-L_{i} C_{2 i}\right) e_{i}+K_{i} \sum_{j \in \mathbf{V}_{i}}\left(e_{j}-e_{i}\right)-\left[\begin{array}{ll}
I & 0
\end{array}\right] \omega_{i} \\
& +\left(B_{2}-L_{i} D_{2 i}\right) \xi-L_{i} \bar{D}_{2 i} \xi_{i}+\nu_{i}, \quad e_{i}(0)=x_{0} \\
\dot{\omega}_{i} & =\Omega_{i} \omega_{i}+\Gamma_{i} \nu_{i} \quad \omega_{i}(0)=0
\end{aligned}
$$

The system (14) equipped with the outputs (12), (13) is an uncertain system governed by $L_{2}$-integrable inputs $\xi, \xi_{i}$ and $\nu_{i}$. Each such system is interconnected with its neighbours via inputs $e_{j}$, and the collection of all such systems represents a large-scale system. We propose the following distributed $H_{\infty}$ observer for this large-scale system which utilizes the outputs 12, 13 to obtain estimates of $e_{i}$ and $\omega_{i}$ while attenuating the disturbances $\xi, \xi_{i}$ and $\nu_{i}$, 


$$
\begin{aligned}
& i=1, \ldots, N: \\
& \dot{\hat{e}}_{i}=\left(A-L_{i} C_{2 i}\right) \hat{e}_{i}+K_{i} \sum_{j \in \mathbf{V}_{i}}\left(\hat{e}_{j}-\hat{e}_{i}\right)-[I 0] \hat{\omega}_{i} \\
& \quad+F_{i}\left(\zeta_{i}-C_{2 i} \hat{e}_{i}\right)+H_{i}\left(\bar{\zeta}_{i}+\sum_{j \in \mathbf{V}_{i}}\left(\hat{e}_{j}-\hat{e}_{i}\right)\right), \\
& \dot{\hat{\omega}}_{i}=\Omega_{i} \hat{\omega}_{i}+F_{i}^{\eta}\left(\zeta_{i}-C_{2 i} \hat{e}_{i}\right)+H_{i}^{\eta}\left(\bar{\zeta}_{i}+\sum_{j \in \mathbf{V}_{i}}\left(\hat{e}_{j}-\hat{e}_{i}\right)\right), \\
& \quad \hat{e}_{i}(0)=0, \quad \hat{\omega}_{i}(0)=0 .
\end{aligned}
$$

The coefficients $F_{i}, H_{i}, F_{i}^{\eta}, H_{i}^{\eta}$ are to be found in such a way that $\hat{\eta}_{i}: \hat{\eta}_{i}=\left[\begin{array}{ll}I & 0\end{array}\right] \hat{\omega}_{i}$ tracks the output $\eta_{i}$ of the auxiliary system (7). Then, since according to the definition of the auxiliary signal $\eta_{i}$, this signal represents $f_{i}$ asymptotically, we propose using $\hat{\eta}_{i}$ as a residual variable indicating whether the attack is taking place.

To formalize the above idea, introduce the error vectors $z_{i}=e_{i}-\hat{e}_{i}, \delta_{i}=\omega_{i}-\hat{\omega}_{i}$. Using the extended system model (14) and the corresponding observer (15), the evolution of these error vectors is governed by the following equations

$$
\begin{aligned}
\dot{z}_{i} & =\left(A-L_{i} C_{2 i}\right) z_{i}+K_{i} \sum_{j \in \mathbf{V}_{i}}\left(z_{j}-z_{i}\right)-\left[\begin{array}{ll}
I & 0
\end{array}\right] \delta_{i} \\
& -F_{i} C_{2 i} z_{i}+H_{i} \sum_{j \in \mathbf{V}_{i}}\left(z_{j}-z_{i}\right) \\
& +\left(B_{2}-L_{i} D_{2 i}\right) \xi-L_{i} \bar{D}_{2 i} \xi_{i}+\nu_{i} \\
& -F_{i} D_{2 i} \xi-F_{i} \bar{D}_{2 i} \xi_{i}, \quad z_{i}(0)=x_{0}, \\
\dot{\delta}_{i} & =\Omega_{i} \delta_{i}-F_{i}^{\eta} C_{2 i} z_{i}+H_{i}^{\eta} \sum_{j \in \mathbf{V}_{i}}\left(z_{j}-z_{i}\right) \\
& -F_{i}^{\eta} D_{2 i} \xi-F_{i}^{\eta} \bar{D}_{2 i} \xi_{i}+\Gamma_{i} \nu_{i}, \quad \delta_{i}(0)=0 .
\end{aligned}
$$

Note that we can introduce new variables $\tilde{L}_{i}=L_{i}+F_{i}$, $\tilde{K}_{i}=K_{i}+H_{i}$, and re-write (16) as

$$
\begin{aligned}
\dot{z}_{i} & =\left(A-\tilde{L}_{i} C_{2 i}\right) z_{i}+\tilde{K}_{i} \sum_{j \in \mathbf{V}_{i}}\left(z_{j}-z_{i}\right)-\left[\begin{array}{ll}
I & 0
\end{array}\right] \delta_{i} \\
& +\left(B_{2}-\tilde{L}_{i} D_{2 i}\right) \xi-\tilde{L}_{i} \bar{D}_{2 i} \xi_{i}+\nu_{i}, \quad z_{i}(0)=x_{0}, \\
\dot{\delta}_{i} & =\Omega_{i} \delta_{i}-F_{i}^{\eta} C_{2 i} z_{i}+H_{i}^{\eta} \sum_{j \in \mathbf{V}_{i}}\left(z_{j}-z_{i}\right) \\
& -F_{i}^{\eta} D_{2 i} \xi-F_{i}^{\eta} \bar{D}_{2 i} \xi_{i}+\Gamma_{i} \nu_{i}, \quad \delta_{i}(0)=0 .
\end{aligned}
$$

Problem 1 (The $H_{\infty}$ detector design problem): The distributed attack detection problem under consideration in this paper is to determine $\tilde{L}_{i}, \tilde{K}_{i}, F_{i}^{\eta}, H_{i}^{\eta}$ such that the following conditions hold:

(i) The large-scale system 177 is internally stable. Equivalently, the disturbance and attack-free large-scale system

$$
\begin{gathered}
\dot{z}_{i}=\left(A-\tilde{L}_{i} C_{2 i}\right) z_{i}+\tilde{K}_{i} \sum_{j \in \mathbf{V}_{i}}\left(z_{j}-z_{i}\right)-\left[\begin{array}{ll}
I & 0
\end{array}\right] \delta_{i} \\
\dot{\delta}_{i}=\Omega_{i} \delta_{i}-F_{i}^{\eta} C_{2 i} z_{i}+H_{i}^{\eta} \sum_{j \in \mathbf{V}_{i}}\left(z_{j}-z_{i}\right) \\
z_{i}(0)=x_{0}, \quad \delta_{i}(0)=0
\end{gathered}
$$

must be asymptotically stable. (ii) In the presence of disturbances and attack signals, all from the class of $L_{2}$-integrable signals, the system (17) achieves a guaranteed level of $H_{\infty}$ filtering performance:

$$
\sup _{x_{0}, \mathbf{w} \neq 0} \frac{\int_{0}^{\infty} \sum_{i=1}^{N}\left(\delta_{i}^{\prime} Q_{i} \delta_{i}+z_{i}^{\prime} \bar{Q}_{i} z_{i}\right) d t}{\left\|x_{0}\right\|_{P}^{2}+\sum_{i=1}^{N}\left\|\mathbf{w}_{i}\right\|_{2}^{2}} \leq \gamma^{2},
$$

where $Q_{i}=Q_{i}^{\prime}>0, \bar{Q}_{i}=\bar{Q}_{i}^{\prime} \geq 0$ are given matrices, $\left\|x_{0}\right\|_{P}^{2}=x_{0}^{\prime} P x_{0}, P=P^{\prime}>0$ is a fixed matrix to be determined later, $\mathbf{w}_{i} \triangleq\left[\xi^{\prime}, \xi_{i}^{\prime}, \nu_{i}^{\prime}\right]^{\prime}, \mathbf{w} \triangleq$ $\left[\mathbf{w}_{1}^{\prime}, \ldots, \mathbf{w}_{N}^{\prime}\right]^{\prime}$, and $\gamma>0$ is a constant.

It follows from 19] that each attack detector variable $\hat{\omega}_{i}$ provides an $H_{\infty}$ estimate of $\omega_{i}$. We now show that provided Assumption 11 holds, the output $\hat{\eta}_{i}=\left[\begin{array}{ll}I & 0\end{array}\right] \hat{\omega}_{i}$ of the observer (15) converges to $f_{i}$, and hence it can be used as a residual indicator of attack.

Lemma 1: Suppose Assumption 1 holds and the observer network (4) is such that the disturbance and attack-free largescale system (18) is asymptotically stable, and also 190 holds with $\bar{Q}_{i}>0$. Then $\left\|\hat{\eta}_{i}-f_{i}\right\| \rightarrow 0$ as $t \rightarrow \infty$ for all $f_{i}$ that have a finite limit at $\infty$.

Note that 19) with $\bar{Q}_{i}>0$ requires the observer to ensure disturbance attenuation with respect to both $\delta_{i}$ and $z_{i}$, even though only the variable $\delta_{i}$ captures the tracking error of interest. When $\bar{Q}_{i}=0$ and condition (19) reduces to a weaker condition we can guarantee that $\hat{\eta}_{i}$ converges to $f_{i}$ in $L_{2}$ sense, even when $f_{i}$ does not have a finite limit at $\infty$.

Lemma 2: Suppose Assumption 1 holds and the observer network (4) is such that the disturbance and attack-free largescale system (18) is asymptotically stable, and also condition (19) holds with $\bar{Q}_{i}=0$,

$$
\sup _{x_{0}, \mathbf{w} \neq 0} \frac{\int_{0}^{\infty} \sum_{i=1}^{N} \delta_{i}^{\prime} Q_{i} \delta_{i} d t}{\left\|x_{0}\right\|_{P}^{2}+\sum_{i=1}^{N}\left\|\mathbf{w}_{i}\right\|_{2}^{2}} \leq \gamma^{2} .
$$

Then $\sum_{i=1}^{N} \int_{0}^{\infty}\left\|\hat{\eta}_{i}-f_{i}\right\|^{2} d t<\infty$.

It is worth noting that the system (15) is governed by the outputs of the observer network (4); therefore it can be implemented to monitor the health of the network. We explain in the next section how to design $\tilde{L}_{i}, \tilde{K}_{i}, F_{i}^{\eta}$, and $H_{i}^{\eta}$ such that the above conditions hold.

\section{ATTACK DETECTOR DESIGN}

Problem 1 belongs to the class of distributed stabilization by output injection problems. References [4], [16], [18] developed a vector dissipativity approach to solve this class of problems which will be applied here as well. For each node $i$, consider a candidate storage function $V_{i}\left(z_{i}, \delta_{i}\right)=$ $\left[\begin{array}{ll}z_{i}^{\prime} & \delta_{i}^{\prime}\end{array}\right] X_{i}\left[\begin{array}{ll}z_{i}^{\prime} & \delta_{i}^{\prime}\end{array}\right]^{\prime}$, where $X_{i}=X_{i}^{\prime}>0$. The following vector dissipation inequality is instrumental in proving input tracking properties of the distributed attack detector (15):

$$
\dot{V}_{i}+2 \alpha_{i} V_{i}+\delta_{i}^{\prime} Q_{i} \delta_{i}+z_{i}^{\prime} \bar{Q}_{i} z_{i} \leq \sum_{j \in \mathbf{V}_{j}} \pi_{j} V_{j}+\gamma^{2}\left\|\mathbf{w}_{i}\right\|^{2},
$$


where $\pi_{i}, \pi_{j}$ are constants selected so that the matrix

$$
\left[\begin{array}{cccc}
-2 \alpha_{1} & \pi_{2} \mathbf{a}_{12} & \ldots & \pi_{N} \mathbf{a}_{1 N} \\
\pi_{1} \mathbf{a}_{21} & -2 \alpha_{2} & \ldots & \pi_{N} \mathbf{a}_{2 N} \\
\vdots & \vdots & \ddots & \vdots \\
\pi_{1} \mathbf{a}_{N 1} & \pi_{2} \mathbf{a}_{N 2} & \ldots & -2 \alpha_{N}
\end{array}\right]
$$

is diagonally dominant (and therefore it is Hurwitz [11]); here, $\mathbf{a}_{i j}$ is the element of the adjacency matrix of the graph $\mathrm{G}$. Indeed adding the inequalities (21) will result in

$$
\begin{aligned}
\sum_{i=1}^{N} \dot{V}_{i} & +\sum_{i=1}^{N}\left(\delta_{i}^{\prime} Q_{i} \delta_{i}+z_{i}^{\prime} \bar{Q}_{i} z_{i}\right) \\
\leq & \max \left\{-2 \alpha_{1}+q_{1} \pi_{1}, \ldots,-2 \alpha_{N}+q_{N} \pi_{N}\right\} \sum_{i=1}^{N} V_{i} \\
& +\gamma^{2} \sum_{i=1}^{N}\left(\|\xi\|^{2}+\left\|\xi_{i}\right\|^{2}+\left\|\nu_{i}\right\|^{2}\right) .
\end{aligned}
$$

Selecting $\pi_{i}<\frac{2 \alpha_{i}}{q_{i}}$ and letting $\varepsilon=\min \left\{2 \alpha_{1}-\right.$ $\left.q_{1} \pi_{1}, \ldots, 2 \alpha_{N}-q_{N} \pi_{N}\right\}>0, V=\sum_{i=1}^{N} V_{i}$, we then have

$$
\begin{aligned}
& \dot{V}+\sum_{i=1}^{N}\left(\delta_{i}^{\prime} Q_{i} \delta_{i}+z_{i}^{\prime} \bar{Q}_{i} z_{i}\right) \leq \\
& \quad-\varepsilon V+\gamma^{2} \sum_{i=1}^{N}\left(\|\xi\|^{2}+\left\|\xi_{i}\right\|^{2}+\left\|\nu_{i}\right\|^{2}\right) .
\end{aligned}
$$

This implies that when $\xi=0$ and $f_{i}=0, \xi_{i}=0 \forall i$, then

$$
\dot{V}<-\varepsilon V
$$

and provided $X_{i}>0$, we have $z_{i} \rightarrow 0, \delta_{i} \rightarrow 0$ exponentially. That is, condition (i) of Problem 1 is established.

Also, when at least one of the signals $\xi, \xi_{i}$ or $f_{i}$ is not equal to zero (the latter is equivalent to $\nu_{i} \not \equiv 0$ ), then it follows from (22) that

$$
\begin{aligned}
& \sum_{i=1}^{N} \int_{0}^{T}\left(\delta_{i}^{\prime} Q_{i} \delta_{i}+z_{i}^{\prime} \bar{Q}_{i} z_{i}\right) d t \leq \sum_{i=1}^{N}\left[V_{i}\left(z_{i}(0), \delta_{i}(0)\right)\right. \\
& \left.\quad+\gamma^{2} \int_{0}^{T}\left(\|\xi\|^{2}+\left\|\xi_{i}\right\|^{2}+\left\|\nu_{i}\right\|^{2}\right) d t\right] .
\end{aligned}
$$

Note that $V_{i}\left(z_{i}(0), \delta_{i}(0)\right)=x_{0}^{\prime} X_{i}^{11} x_{0}$, where $X_{i}^{11}$ is the upper left block in the partition of $X_{i}$ compatible with the dimensions of $z_{i}$ and $\delta_{i}$. Hence (19) also holds with $P=$ $\gamma^{-2} \sum_{i=1}^{N} X_{i}^{11}$. It follows from this discussion that condition (21) ensures satisfaction of the conditions of Lemma 1 Therefore, to ensure that the distributed observer (15) can track the attack input $f_{i}$ we need to determine coefficients $\tilde{L}_{i}, \tilde{K}_{i}, F_{i}^{\eta}$, and $H_{i}^{\eta}$ for it so that 21) is satisfied.

To present conditions under which (21) holds, introduce the notation

$$
\begin{aligned}
& A_{i}^{\mu}=\left[\begin{array}{cc}
A & -\left[\begin{array}{ll}
I & 0
\end{array}\right] \\
0 & \Omega_{i}
\end{array}\right], \quad B_{1}^{\mu}=\left[\begin{array}{c}
I \\
\Gamma_{i}
\end{array}\right], \quad B_{2}^{\mu}=\left[\begin{array}{cc}
-B_{2} & 0 \\
0 & 0
\end{array}\right], \\
& D_{2 i}^{\mu}=\left[\begin{array}{ll}
D_{2 i} & \bar{D}_{2 i}
\end{array}\right], \quad C_{2 i}^{\mu}=\left[\begin{array}{ll}
C_{2 i} & 0
\end{array}\right], \quad H^{\mu}=\left[\begin{array}{ll}
I & 0
\end{array}\right], \\
& L_{i}^{\mu}=\left[\begin{array}{c}
\tilde{L}_{i} \\
F_{i}^{\eta}
\end{array}\right], \quad K_{i}^{\mu}=\left[\begin{array}{c}
\tilde{K}_{i} \\
H_{i}^{\eta}
\end{array}\right] .
\end{aligned}
$$

Suppose $D_{2 i}$ and $\bar{D}_{2 i}$ satisfy the condition

$$
E_{2 i} \triangleq D_{2 i}^{\mu}\left(D_{2 i}^{\mu}\right)^{\prime}=D_{2 i} D_{2 i}^{\prime}+\bar{D}_{2 i} \bar{D}_{2 i}^{\prime}>0 .
$$

The above assumption on $E_{2 i}$ is a standard assumption made in nonsingular $H_{\infty}$ control problems [1].

Now let us introduce the matrix

$$
Q_{i}^{\mu}=\left[\begin{array}{cc}
\bar{Q}_{i} & 0 \\
0 & Q_{i}
\end{array}\right],
$$

where $Q_{i}=Q_{i}^{\prime}>0$. Also, $\bar{Q}_{i}=\bar{Q}_{i}^{\prime}$ is selected to be positive definite when the aim is to design an attack observer to achieve asymptotic tracking of attack inputs. If $L_{2}$ tracking is acceptable, one can let $\bar{Q}_{i}=0$. Given $\alpha_{i}>0$, define $\pi_{i}=\frac{2 \alpha_{i}}{q_{i}+1}$, where $q_{i}$ is the out-degree of the graph node $i$. Clearly $\pi_{i}=\frac{2 \alpha_{i}}{q_{i}+1}<\frac{2 \alpha_{i}}{q_{i}}$.

Theorem 1: Suppose Assumption 1 holds and the digraph $\mathbf{G}$, the matrices $Q_{i}=Q_{i}^{\prime}>0, \bar{Q}_{i}=\bar{Q}_{i}^{\prime}>0, i=1, \cdots, 6$ and the constants $\alpha_{i}>0, i=1, \cdots, N$ are such that the coupled linear matrix inequalities in 27) (on the next page) with respect to the variables $X_{i}=X_{i}^{\prime}>0$ and $M_{i}, i=$ $1, \cdots, N$ are feasible. Then choosing

$$
\begin{aligned}
K_{i}^{\mu} & =-X_{i}^{-1} M_{i}, \\
L_{i}^{\mu} & =\left(\gamma^{2} X_{i}^{-1}\left(C_{2 i}^{\mu}\right)^{\prime}-B_{2}^{\mu}\left(D_{2 i}^{\mu}\right)^{\prime}\right) E_{2 i}^{-1}
\end{aligned}
$$

ensures that the condition 21) holds.

Combined with Lemma 1 or Lemma 2] this theorem provides a complete result on the design of biasing attack detectors for the distributed observer (4).

\section{CONCLUSION}

The paper is concerned with the problem of distributed attack detection is sensor networks. We consider a group of consensus-based distributed estimators and assume that the estimator dynamics are under attack. Then we propose a distributed $H_{\infty}$ attack detector which allows for a broad range of uncertainty in the sensors and the plant model, as well as a quite broad range of bias attack inputs, and show that the proposed attack detector can track individual attack inputs at different sensors. A possible future direction is to construct a compensator to cancel the detected attack in the system.

\section{ACKNOWLEDGEMENT}

The authors thank G. Seyboth for providing his paper [12].

\section{REFERENCES}

[1] T. Başar, and P. Bernhard. $H_{\infty}$ optimal control and related minimax design problems: a dynamic game approach. Springer Science \& Business Media, 2008.

[2] R. M. Ferrari, T. Parisini, M. M. Polycarpou. Distributed fault detection and isolation of large-scale discrete-time nonlinear systems: An adaptive approximation approach. IEEE Transactions on Automatic Control. 57(2): 275-90m 2012.

[3] X. Ge, Q. L. Han, and X. Jiang. Distributed fault detection for sensor networks with Markovian sensing topology. In Proc. American Control Conference (ACC), pages 3555-3560, 2013.

[4] W. M. Haddad, V. Chellaboina, and S. G. Nersesov. Vector dissipativity theory and stability of feedback interconnections for large-scale non-linear dynamical systems. Int. J. Contr., 77(10):907-919, 2004. 


$$
\begin{aligned}
& {\left[\begin{array}{cccccc}
S_{i} & X_{i} B_{1}^{\mu} & X_{i} B_{2}^{\mu}\left(I-\left(D_{2 i}^{\mu}\right)^{\prime} E_{2 i}^{-1} D_{2 i}^{\mu}\right) & -M_{i} H^{\mu} & \ldots & -M_{i} H^{\mu} \\
\left(B_{1}^{\mu}\right)^{\prime} X_{i} & -\gamma^{2} I & 0 & 0 & \ldots & 0 \\
\left(I-\left(D_{2 i}^{\mu}\right)^{\prime} E_{2 i}^{-1} D_{2 i}^{\mu}\right)\left(B_{2}^{\mu}\right)^{\prime} X_{i} & 0 & -\gamma^{2} I & 0 & \ldots & 0 \\
-\left(H^{\mu}\right)^{\prime} M_{i}^{\prime} & 0 & 0 & -\frac{2 \alpha_{j_{1}}}{q_{j_{1}}+1} X_{j_{1}} & \ldots & 0 \\
\vdots & \vdots & \vdots & \ddots & \vdots & \vdots \\
-\left(H^{\mu}\right)^{\prime} M_{i}^{\prime} & 0 & 0 & 0 & \ldots & -\frac{2 \alpha_{j_{p_{i}}}}{q_{j_{p_{i}}+1}+1} X_{j_{p_{i}}}
\end{array}\right]<0,} \\
& S_{i}=X_{i}\left(A_{i}^{\mu}+\alpha_{i} I+B_{2}^{\mu}\left(D_{2 i}^{\mu}\right)^{\prime} E_{2 i}^{-1} C_{2 i}^{\mu}\right)+\left(A_{i}^{\mu}+\alpha_{i} I+B_{2}^{\mu}\left(D_{2 i}^{\mu}\right)^{\prime} E_{2 i}^{-1} C_{2 i}^{\mu}\right)^{\prime} X_{i} \\
& +p_{i} M_{i} H^{\mu}+p_{i}\left(H^{\mu}\right)^{\prime} M_{i}^{\prime}+Q_{i}^{\mu}-\gamma^{2}\left(C_{2 i}^{\mu}\right)^{\prime} E_{2 i}^{-1} C_{2 i}^{\mu} \text {. }
\end{aligned}
$$

[5] X. He, Z. Wang, Y. D. Ji, and D. H. Zhou. Robust fault detection for networked systems with distributed sensors. IEEE Transactions on Aerospace and Electronic Systems, 47(1):166-177, 2011.

[6] J. Löfberg. YALMIP: a toolbox for modeling and optimization in MATLAB. In Proc. CACSD Conference, Taipei, Taiwan, pages $284-$ 289, 2004.

[7] R. Olfati-Saber. Distributed Kalman filtering for sensor networks. In Proc. 46th IEEE CDC, pages 5492-5498, 2007.

[8] F. Pasqualetti, A. Bicchi, and F. Bullo. Consensus computation in unreliable networks: A system theoretic approach. IEEE Trans. Automat. Contr., 57:90-104, 2012.

[9] F. Pasqualetti, F. Dorfler, and F. Bullo. Attack detection and identification in cyber-physical systems. IEEE Tran. Automat. Contr., 58:27152729, 2013.

[10] F. Pasqualetti, F. Dorfler, and F. Bullo. Control-theoretic methods for cyberphysical security: Geometric principles for optimal cross-layer resilient control systems. IEEE Control Systems, 35:110-127, 2015.

[11] D. D. Siljak. Large-scale dynamic systems: stability and structure. North-Holland, 1978.

[12] G. S. Seyboth and F. Allgower. Output synchronization of linear multiagent systems under constant disturbances via distributed integral action. In Proc. American Control Conference (ACC), pages 62-67, 2015.

[13] A. Teixeira, I. Shames, H. Sandberg, and K. H. Johansson. Distributed fault detection and isolation resilient to network model uncertainties. IEEE Transactions on Cybernetics, 44(11):2024 - 2037, 2014.

[14] A. Teixeira, I. Shames, H. Sandberg, and K. H. Johansson. A secure control framework for resource-limited adversaries. Automatica, 51: $135-148,2015$.

[15] B. Shen, Z. Wang, and Y. S. Hung. Distributed $H_{\infty}$-consensus filtering in sensor networks with multiple missing measurements: The finitehorizon case. Automatica, 46(10):1682 - 1688, 2010.

[16] V. Ugrinovskii. Distributed robust filtering with $H_{\infty}$ consensus of estimates. Automatica, 47(1):1 - 13, 2011.

[17] V. Ugrinovskii. Gain-scheduled synchronization of parameter varying systems via relative $H_{\infty}$ consensus with application to synchronization of uncertain bilinear systems. Automatica, 50(11):2880-2887, 2014. arXiv:1406.5622 [cs.SY].

[18] V. Ugrinovskii and C. Langbort. Distributed $H_{\infty}$ consensus-based estimation of uncertain systems via dissipativity theory. IET Control Theory \& App., 5(12):1458-1469, 2011. 